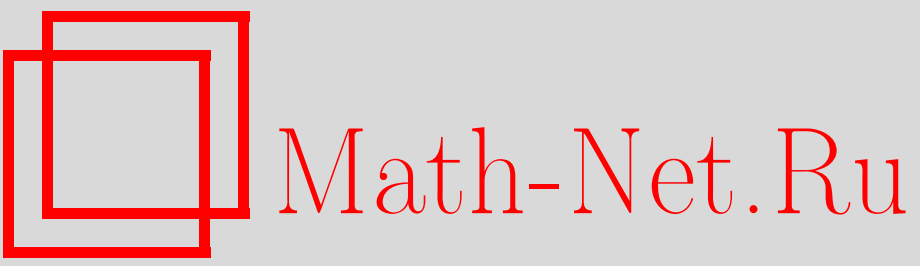

В. В. Махнач, О квазианапольном взаимодействии заряженных фермионов, TMФ, 2004, том 139, номер 3, 423-428

DOI: https://doi.org/10.4213/tmf68

Использование Общероссийского математического портала Math-Net.Ru подразумевает, что вы прочитали и согласны с пользовательским соглашением

http://www.mathnet.ru/rus/agreement

Параметры загрузки:

IP: 35.174 .16 .151

26 апреля 2023 г., 02:19:01 
ТЕОРЕТИЧЕСКАЯ

И МАТЕМАТИЧЕСКАЯ

ФИЗИКА

Том 139, № 3

июнь, 2004

(C) 2004 г.

B.В. Махнач*

\section{О КВАЗИАНАПОЛЬНОМ ВЗАИМОДЕЙСТВИИ ЗАРЯЖЕННЫХ ФЕРМИОНОВ}

Получено аналитическое выражение для полного сечения реакции $e^{-} e^{+} \rightarrow l^{-} l^{+}$ $(l=\mu, \tau)$ с учетом возможного проявления квазианапольного взаимодействия. Приведены ограничения для численного значения параметра взаимодействия из данных реакции $e^{-} e^{+} \rightarrow \mu^{-} \mu^{+}$в области энергий ниже $Z^{0}$-пика.

Ключевые слова: слабые и электромагнитные взаимодействия лептонов, нейтральные токи.

\section{1. ВВЕДЕНИЕ}

В настоящее время стандартная модель электрослабых взаимодействий (СМ) хорошо описывает экспериментальную ситуацию для слабых и электромагнитных взаимодействий элементарных частищ. Тем не менее сушествуют причины, указываюшие на необходимость поисков выходов за ее пределы. Анапольное взаимодействие элементарных частиц - одна из них.

Зельдовичем было показано [1], что вследствие несохранения четности в слабых процессах при взаимодействии фермионов $(s=1 / 2)$ с виртуальным электромагнитным полем необходимо учитывать вклад вида $\vec{\sigma} \operatorname{rot} \vec{H} \sim(\vec{\sigma} \cdot \vec{j})$, где $\vec{j}$ - вектор плотности тока, создаюшего поле $\vec{H}$, а $\vec{\sigma}-$ спин частицы. Возникаюший в этом случае момент был назван анапольным (AM). Отметим также, что описание этого свойства в рамках релятивистского подхода было дано в работе [2].

Был выполнен ряд работ по исследованию АМ элементарных частиц. В реакциях как между заряженными, так и нейтральными фермионами АМ учитывался посредством учета электрослабых поправок, и для электрона он был вычислен в работе [3]. Однако затем в статьях [4], [5] было показано, что поскольку это свойство в рамках СМ является калибровочно-зависимым, то нельзя выделить его физический вклад. Показано было также, что АМ следует рассматривать только совместно с другими процессами, обусловленными нейтральными токами. Выделение же АМ как физически наблюдаемого свойства возможно только для составных фермионных систем. Так, АМ атома содержит калибровочно-инвариантный член, величина которого растет как $A^{2 / 3}$, где $A$ - атомный номер [6].

* Институт физики им. Б. И. Степанова НАН Беларуси, Минск, Беларусь. Email: makh@dragon.bas-net.by 
Однако следует также отметить, что если определять АМ свободного фермиона как его аксиально-векторное контактное взаимодействие с внешним электромагнитным полем, то можно получить для него калибровочно-инвариантное выражение [7].

Вне рамок СМ в работе [8] рассмотрена идея о возможности сушествования нового взаимодействия между бозонами и заряженными фермионами, не сохраняюшего отдельно $C$ - и $P$-четность, однако $C P$-инвариантного. Этот подход заключается в идее рассмотрения элементарных частиц как составных объектов. Термин "квазианапольное взаимодействие" (КАВ) выделяет эту идею в феноменологическом плане, а данная работа представляет собой дальнейшее следование ей.

Весомым доводом в пользу продолжения исследований в этом направлении являются выполненные успешные эксперименты по измерению АМ ядра атома ${ }^{133} \mathrm{Cs}[9]$ и протона [10].

\section{2. "КВАЗИАНАПОЛЬНОЕ" СЕЧЕНИЕ}

Запишем лагранжиан взаимодействия фермионного поля $(f)$ с электромагнитным $\left(A_{\mu}\right)$ и массивным нейтральным $\left(Z_{\mu}^{0}\right)$ полями в наших обозначениях в следуюшем виде:

$$
\mathcal{L}=i e\left(Q_{f} \bar{f} \gamma_{\mu} f A_{\mu}+\frac{1}{2 \sin 2 \theta_{\mathrm{W}}} \bar{f} \gamma_{\mu}\left(g_{\mathrm{V}}^{f}+g_{\mathrm{A}}^{f} \gamma_{5}\right) f Z_{\mu}^{0}\right),
$$

где $e$ - заряд протона, $Q_{f}$ - заряд фермионного поля в единицах заряда $e, \theta_{\mathrm{W}}$ - угол Вайнберга, $g_{\mathrm{V}}^{f}$ и $g_{\mathrm{A}}^{f}-$ векторная и аксиальная фермионные константы, соответственно:

$$
\begin{aligned}
& g_{\mathrm{V}}^{f}=2\left(t_{3 L}(f)-2 Q_{f} \sin ^{2} \theta_{\mathrm{W}}\right), \\
& g_{\mathrm{A}}^{f}=2\left(t_{3 L}(f)\right) .
\end{aligned}
$$

Здесь $t_{3 L}(f)$ - третья проекция слабого изотопического спина фермиона; $t_{3 L}(f)=-1 / 2$ для полей $f: e^{-}, \mu^{-}, \tau^{-}$.

Общее выражение для калибровочно-инвариантной вершины взаимодействия фермиона (спин $1 / 2)$ с нейтральным векторным бозоном имеет вид

$$
\Gamma_{\nu}=Q \gamma_{\nu}+\mu \sigma_{\nu \rho} q_{\rho}+\kappa\left(q^{2} \delta_{\nu \rho}-q_{\nu} q_{\rho}\right) \gamma_{\rho} \gamma_{5}+D \sigma_{\nu \rho} \gamma_{5} q_{\rho}
$$

где $Q, \mu, D$ характеризуют электрический, аномальный магнитный и дипольный электрический заряды, соответственно; член с коэффициентом $\kappa$ представляет КАВ. Соответственно $\gamma_{\mu}$ - матрицы Дирака, $\gamma_{5}=\gamma_{1} \gamma_{2} \gamma_{3} \gamma_{4} ; \sigma_{\mu \nu}=i\left(\gamma_{\mu} \gamma_{\nu}-\gamma_{\nu} \gamma_{\mu}\right) / 2 ; q$-переданный импульс. С целью получения именно феноменологических результатов, которые позволят оценить возможное проявление К АВ, в выражении (3) будут учтены лишь первое и третье слагаемые.

Для анализа КАВ рассмотрим реакцию $e \bar{e} \rightarrow f \bar{f}$ (где $f: \mu, \tau)$. Подобный анализ был выполнен в работе [8]. Однако теперь помимо вершины, соответствуюшей испусканию 
виртуального $\gamma$-кванта, модифицируем $Z^{0}$-вершину, что соответствует КАВ электронного (фермионного) тока с полем $Z^{0}$-бозона. Модифицированный таким образом лагранжиан запишем в следуюшем виде:

$$
\begin{aligned}
\mathcal{L}= & i e\left[\bar{f}\left(Q \gamma_{\mu}+\kappa_{f}^{\gamma}\left(q^{2} \delta_{\mu \rho}-q_{\mu} q_{\rho}\right) \gamma_{\rho} \gamma_{5}\right) f A_{\mu}+\right. \\
& \left.+\frac{1}{2 \sin 2 \theta_{\mathrm{W}}} \bar{f}\left(\gamma_{\mu}+\kappa_{f}^{Z}\left(q^{2} \delta_{\mu \rho}-q_{\mu} q_{\rho}\right) \gamma_{\rho} \gamma_{5}\right)\left(g_{\mathrm{V}}^{f}+g_{\mathrm{A}}^{f} \gamma_{5}\right) f Z_{\mu}^{0}\right] .
\end{aligned}
$$

Поскольку численная оценка параметра КАВ будет проведена для реакций в диапазоне энергий, значительно превышаюших массы участвуюших фермионов, пренебрегаем в дальнейших выкладках "массовым" членом $\left(q_{\nu} q_{\rho} \gamma_{\rho} \gamma_{5}\right)$ при коэффициенте $\kappa$.

Далее нижние индексы у $\kappa(e$ и $f)$ будут обозначать электронную или фермионную вершины, соответственно, а верхние ( $\gamma$ и $Z)$ - поле виртуального переносчика взаимодействия (электромагнитное или нейтральное векторное).

Выражение для дифференциального сечения реакции $e \bar{e} \rightarrow f \bar{f}$ с учетом квазианапольных добавок можно записать в виде

$$
\frac{d \sigma(e \bar{e} \rightarrow \mu \bar{\mu}, \tau \bar{\tau})}{d \Omega}=F_{A}\left(1+\cos ^{2} \theta\right)+2 F_{B} \cos \theta
$$

где

$$
\begin{aligned}
F_{(A, B)}= & \frac{1}{4 S} \alpha^{2} Q_{f}^{2}(A, B)-2 Q_{f} \frac{\alpha}{32 \pi} \frac{G_{\mathrm{F}}}{\sqrt{2}}\left[\left(\frac{S}{M_{Z}^{2}}-1\right)+\frac{\Gamma_{Z}^{2}}{S-M_{Z}^{2}}\right]^{-1}\left(A^{\prime}, B^{\prime}\right)+ \\
& +\frac{1}{256 \pi^{2}}\left(\frac{G_{\mathrm{F}}}{\sqrt{2}}\right)^{2} S\left[\left(\frac{S}{M_{Z}^{2}}-1\right)^{2}+\frac{\Gamma_{Z}^{2}}{M_{Z}^{2}}\right]^{-1}\left(A^{\prime \prime}, B^{\prime \prime}\right)
\end{aligned}
$$

$\left(G_{\mathrm{F}}\right.$ - константа Ферми), а коэффициенты $A^{(),\left(^{\prime}\right),\left(^{\prime \prime}\right)}$ и $B^{(),\left(^{\prime}\right),\left(^{\prime \prime}\right)}$ выражены через параметры теории:

$$
\begin{aligned}
A=1 & +\left(\kappa_{e}^{\gamma}\right)^{2} S^{2}+\left(\kappa_{f}^{\gamma}\right)^{2} S^{2}+\left(\kappa_{e}^{\gamma}\right)^{2}\left(\kappa_{f}^{\gamma}\right)^{2} S^{4}, \\
A^{\prime}= & g_{\mathrm{V}}^{f} g_{\mathrm{V}}^{e}\left(1+\kappa_{e}^{Z} \kappa_{e}^{\gamma} S^{2}+\kappa_{f}^{Z} \kappa_{f}^{\gamma} S^{2}+\kappa_{e}^{Z} \kappa_{e}^{\gamma} \kappa_{f}^{Z} \kappa_{f}^{\gamma} S^{4}\right)+ \\
& +g_{\mathrm{A}}^{e} g_{\mathrm{V}}^{f}\left(1+\kappa_{f}^{Z} \kappa_{f}^{\gamma} S^{2}\right)\left(\kappa_{e}^{\gamma} S+\kappa_{e}^{Z} S\right)+ \\
& +g_{\mathrm{A}}^{f} g_{\mathrm{V}}^{e}\left(1+\kappa_{e}^{Z} \kappa_{e}^{\gamma} S^{2}\right)\left(\kappa_{f}^{\gamma} S+\kappa_{f}^{Z} S\right)+ \\
& +g_{\mathrm{A}}^{e} g_{\mathrm{A}}^{f}\left(\kappa_{f}^{\gamma} \kappa_{e}^{\gamma} S^{2}+\kappa_{f}^{\gamma} \kappa_{e}^{Z} S^{2}+\kappa_{f}^{Z} \kappa_{e}^{\gamma} S^{2}+\kappa_{f}^{Z} \kappa_{e}^{Z} S^{2}\right), \\
A^{\prime \prime}= & {\left.\left[\left(g_{\mathrm{V}}^{e}\right)^{2}+\left(g_{\mathrm{A}}^{e}\right)^{2}\right]\left(\left(g_{\mathrm{V}}^{f}\right)^{2}+\left(g_{\mathrm{A}}^{f}\right)^{2}\right)\right] \times } \\
& \times\left[1+\left(\kappa_{e}^{Z}\right)^{2} S^{2}+\left(\kappa_{f}^{Z}\right)^{2} S^{2}+\left(\kappa_{e}^{Z}\right)^{2}\left(\kappa_{f}^{Z}\right)^{2} S^{4}\right]+ \\
& +4 g_{\mathrm{A}}^{e} g_{\mathrm{V}}^{e}\left[\left(g_{\mathrm{V}}^{f}\right)^{2}+\left(g_{\mathrm{A}}^{f}\right)^{2}\right] \kappa_{e}^{Z} S\left[1+\left(\kappa_{f}^{Z}\right)^{2} S^{2}\right]+ \\
& +4 g_{\mathrm{A}}^{f} g_{\mathrm{V}}^{f}\left[\left(g_{\mathrm{V}}^{e}\right)^{2}+\left(g_{\mathrm{A}}^{e}\right)^{2}\right] \kappa_{f}^{Z} S\left[1+\left(\kappa_{e}^{Z}\right)^{2} S^{2}\right]+ \\
& +4 g_{\mathrm{A}}^{f} g_{\mathrm{A}}^{e} g_{\mathrm{V}}^{f} g_{\mathrm{V}}^{e} 4 \kappa_{f}^{Z} \kappa_{e}^{Z} S^{2}, \\
B= & 4 \kappa_{f}^{\gamma} \kappa_{e}^{\gamma} S^{2}, \\
B^{\prime}= & g_{\mathrm{A}}^{f} g_{\mathrm{A}}^{e}\left(1+\kappa_{e}^{Z} \kappa_{e}^{\gamma} S^{2}+\kappa_{f}^{Z} \kappa_{f}^{\gamma} S^{2}+\kappa_{e}^{Z} \kappa_{e}^{\gamma} \kappa_{f}^{Z} \kappa_{f}^{\gamma} S^{4}\right)+
\end{aligned}
$$




$$
\begin{aligned}
& +g_{\mathrm{A}}^{f} g_{\mathrm{V}}^{e}\left(1+\kappa_{f}^{Z} \kappa_{f}^{\gamma} S^{2}\right)\left(\kappa_{e}^{\gamma} S+\kappa_{e}^{Z} S\right)+g_{\mathrm{V}}^{f} g_{\mathrm{A}}^{e}\left(1+\kappa_{e}^{Z} \kappa_{e}^{\gamma} S^{2}\right)\left(\kappa_{f}^{\gamma} S+\kappa_{f}^{Z} S\right)+ \\
& +g_{\mathrm{V}}^{e} g_{\mathrm{V}}^{f}\left(\kappa_{f}^{\gamma} \kappa_{e}^{\gamma} S^{2}+\kappa_{f}^{\gamma} \kappa_{e}^{Z} S^{2}+\kappa_{f}^{Z} \kappa_{e}^{\gamma} S^{2}+\kappa_{f}^{Z} \kappa_{e}^{Z} S^{2}\right), \\
B^{\prime \prime}= & 4 g_{\mathrm{A}}^{f} g_{\mathrm{A}}^{e} g_{\mathrm{V}}^{f} g_{\mathrm{V}}^{e}\left[1+\left(\kappa_{e}^{Z}\right)^{2} S^{2}+\left(\kappa_{f}^{Z}\right)^{2} S^{2}+\left(\kappa_{e}^{Z}\right)^{2}\left(\kappa_{f}^{Z}\right)^{2} S^{4}\right]+ \\
& +4 g_{\mathrm{A}}^{f} g_{\mathrm{V}}^{f}\left[\left(g_{\mathrm{A}}^{e}\right)^{2}+\left(g_{\mathrm{V}}^{e}\right)^{2}\right] \kappa_{e}^{Z} S\left[1+\left(\kappa_{f}^{Z}\right)^{2} S^{2}\right]+ \\
& +4 g_{\mathrm{A}}^{e} g_{\mathrm{V}}^{e}\left[\left(g_{\mathrm{V}}^{f}\right)^{2}+\left(g_{\mathrm{A}}^{f}\right)^{2}\right] \kappa_{f}^{Z} S\left[1+\left(\kappa_{e}^{Z}\right)^{2} S^{2}\right]+ \\
& +\left[\left(g_{\mathrm{V}}^{e}\right)^{2}+\left(g_{\mathrm{A}}^{e}\right)^{2}\right]\left[\left(g_{\mathrm{V}}^{f}\right)^{2}+\left(g_{\mathrm{A}}^{f}\right)^{2}\right] 4 \kappa_{f}^{Z} \kappa_{e}^{Z} S^{2} .
\end{aligned}
$$

Здесь $\alpha$ - постоянная тонкой структуры, $S$ - квадрат суммы начальных и конечных 4 -импульсов в системе центра масс со знаком "минус", $M_{Z}$ - масса, а $\Gamma_{Z}$ - ширина резонансного пика $Z^{0}$-бозона. Для упрощения дальнейшего анализа предполагаем лептонную универсальность КАВ:

$$
\kappa_{f}=\kappa_{e}=\kappa=\kappa^{\gamma}=\kappa^{Z}, \quad g_{\mathrm{V}}^{e}=g_{\mathrm{V}}^{f}=g_{\mathrm{V}}, \quad g_{\mathrm{A}}^{e}=g_{\mathrm{A}}^{f}=g_{\mathrm{A}} .
$$

В итоге имеем следующие выражения для коэффициентов (7):

$$
\begin{aligned}
A= & 1+(\kappa S)^{2}+(\kappa S)^{4}, \\
A^{\prime}= & g_{\mathrm{V}}^{2}+4 g_{\mathrm{A}} g_{\mathrm{V}}(\kappa S)+\left(2 g_{\mathrm{V}}^{2}+4 g_{\mathrm{A}}^{2}\right)(\kappa S)^{2}+4 g_{\mathrm{A}} g_{\mathrm{V}}(\kappa S)^{3}+g_{\mathrm{V}}^{2}(\kappa S)^{4}, \\
A^{\prime \prime}= & \left(g_{\mathrm{V}}^{2}+g_{\mathrm{A}}^{2}\right)^{2}+8 g_{\mathrm{A}} g_{\mathrm{V}}\left(g_{\mathrm{V}}^{2}+g_{\mathrm{A}}^{2}\right) \kappa S+2\left[\left(g_{\mathrm{V}}^{2}+g_{\mathrm{A}}^{2}\right)+8\left(g_{\mathrm{V}} g_{\mathrm{A}}\right)^{2}\right](\kappa S)^{2}+ \\
& +8 g_{\mathrm{A}} g_{\mathrm{V}}\left(g_{\mathrm{V}}^{2}+g_{\mathrm{A}}^{2}\right)(\kappa S)^{3}+\left(g_{\mathrm{V}}^{2}+g_{\mathrm{A}}^{2}\right)^{2}(\kappa S)^{4} . \\
B= & 4(\kappa S)^{2} \\
B^{\prime}= & g_{\mathrm{A}}^{2}+4 g_{\mathrm{A}} g_{\mathrm{V}} \kappa S+\left(2 g_{\mathrm{A}}^{2}+4 g_{\mathrm{V}}^{2}\right)(\kappa S)^{2}+4 g_{\mathrm{A}} g_{\mathrm{V}}(\kappa S)^{3}+g_{\mathrm{A}}^{2}(\kappa S)^{4}, \\
B^{\prime \prime}= & 4 g_{\mathrm{A}}^{2} g_{\mathrm{V}}^{2}+8 g_{\mathrm{A}} g_{\mathrm{V}}\left(g_{\mathrm{A}}^{2}+g_{\mathrm{V}}^{2}\right) \kappa S+4\left[2 g_{\mathrm{A}}^{2} g_{\mathrm{V}}^{2}+\left(g_{\mathrm{A}}^{2}+g_{\mathrm{V}}^{2}\right)^{2}\right](\kappa S)^{2}+ \\
& +8 g_{\mathrm{V}} g_{\mathrm{A}}\left(g_{\mathrm{A}}^{2}+g_{\mathrm{V}}^{2}\right)(\kappa S)^{3}+4 g_{\mathrm{A}} g_{\mathrm{V}}(\kappa S)^{4} . \\
&
\end{aligned}
$$

Для получения полного сечения выполним в (5) интегрирование по телесному углу $d \Omega$. Результат запишем в следуюшем виде:

$$
\begin{aligned}
\sigma= & \frac{4}{3} \pi \alpha^{2} \frac{1}{S}\left(1+2(\kappa S)^{2}+(\kappa S)^{4}\right)+ \\
& +2 \frac{\alpha}{6} \frac{G_{\mathrm{F}} M_{z}^{2}}{\sqrt{2}} \frac{S-M_{Z}^{2}}{\left(S-M_{Z}^{2}\right)^{2}+\left(\Gamma_{Z} M_{Z}\right)^{2}} \times \\
& \times\left[g_{\mathrm{V}}^{2}+\kappa S g_{\mathrm{A}} g_{\mathrm{V}}+(\kappa S)^{2}\left(4 g_{\mathrm{A}}^{2}+2 g_{\mathrm{V}}^{2}\right)+4 g_{\mathrm{A}} g_{\mathrm{V}}(\kappa S)^{3}+g_{\mathrm{V}}^{2}(\kappa S)^{4}\right]+ \\
& +\frac{1}{48 \pi}\left(\frac{G_{\mathrm{F}} M_{z}^{2}}{\sqrt{2}}\right)^{2} \frac{S}{\left(S-M_{Z}^{2}\right)^{2}+\left(\Gamma_{Z} M_{Z}\right)^{2}} \times \\
& \times\left[\left(g_{\mathrm{V}}^{2}+g_{\mathrm{A}}^{2}\right)^{2}\left(1+(\kappa S)^{4}\right)+8 g_{\mathrm{V}} g_{\mathrm{A}}\left(g_{\mathrm{V}}^{2}+g_{\mathrm{A}}^{2}\right)(\kappa S)+\right. \\
& \left.+2(\kappa S)^{2}\left(8 g_{\mathrm{A}}^{2} g_{\mathrm{V}}^{2}+\left(g_{\mathrm{V}}^{2}+g_{\mathrm{A}}^{2}\right)^{2}\right)+(\kappa S)^{3} 8 g_{\mathrm{A}} g_{\mathrm{V}}\left(g_{\mathrm{V}}^{2}+g_{\mathrm{A}}^{2}\right)\right] .
\end{aligned}
$$


В целях удобства проведения дальнейшего анализа перепишем выражение (10) в виде суммы по степеням $\kappa$; введем также следуюшие обозначения:

$$
\begin{aligned}
k & =\frac{G_{\mathrm{F}} M_{Z}^{2}}{2 \sqrt{2} \pi \alpha}, \\
C & =\frac{S\left(S-M_{Z}^{2}\right)}{\left(S-M_{Z}^{2}\right)^{2}+\left(\Gamma_{Z} M_{Z}\right)^{2}}, \\
D & =\frac{S^{2}}{\left(S-M_{Z}^{2}\right)^{2}+\left(\Gamma_{Z} M_{Z}\right)^{2}} .
\end{aligned}
$$

Полное сечение есть

$$
\sigma=\sum_{i=0}^{4} a_{i} \kappa^{i}
$$

а верхний индекс у $\kappa$ - показатель степени. Коэффициенты $a_{i}$ записываются в следующем виде:

$$
\begin{aligned}
& a_{0}=\frac{4}{3} \frac{\pi \alpha^{2}}{S}\left[1+\frac{1}{2} k C g_{\mathrm{V}}^{2}+\frac{1}{16} k^{2} D\left(g_{\mathrm{V}}^{2}+g_{\mathrm{A}}^{2}\right)^{2}\right], \\
& a_{1}=\frac{2}{3} \pi \alpha^{2} k g_{\mathrm{A}} g_{\mathrm{V}}\left[4 C+k D\left(g_{\mathrm{V}}^{2}+g_{\mathrm{A}}^{2}\right)\right], \\
& a_{2}=\frac{4}{3} \pi \alpha^{2} S\left[k C\left(g_{\mathrm{V}}^{2}+2 g_{\mathrm{A}}^{2}\right)+g_{\mathrm{A}} g_{\mathrm{V}} k^{2} D\left(g_{\mathrm{V}}^{2}+g_{\mathrm{A}}^{2}\right)\right], \\
& a_{3}=a_{1} S^{2}, \\
& a_{4}=a_{0} S^{3} .
\end{aligned}
$$

\section{3. ЧИСЛЕННЫЕ ОГРАНИЧЕНИЯ ДЛЯ ПАРАМЕТРА КАВ}

Для получения феноменологических ограничений для параметра КАВ $\kappa$ притягательными являются данные группы $L 3$ [11] для реакции $e^{+} e^{-} \rightarrow \mu^{+} \mu^{-}$, где в энергетической области $60-90$ ГэВ полученные результаты полного сечения отличаются от значений, вычисленных в рамках СМ. Для $\langle\sqrt{S}\rangle \sim 65.8$ ГэВ отличие достигает $2 \sigma$, где $\sigma$ - одно стандартное отклонение. Кроме того, экспериментальные результаты учитывают поправки на излучение фотонов в начальном и конечном состояниях, что также благоприятствует проведению такого анализа.

Используя метод наименьших квадратов и численные значения для $M_{Z}, \Gamma_{Z}$ из работы [12], для шести экспериментальных точек была получена следующая оценка параметра $\mathrm{KAB}$ :

$$
\kappa=6_{-1}^{+20} \times 10^{-6} \Gamma \ni \mathrm{B}^{-2} .
$$

Из безразмерности величины $(\kappa S)$ следует, что параметр вида $1 / \sqrt{\kappa}$ может быть выражен в энергетических единицах в соответствуюших границах:

$$
1 / \sqrt{\kappa}=400_{-200}^{+44} \text { ГэВ. }
$$

Асимметричность ошибки указывает на то, что при уменьшении значения полного сечения реакции ограничение на параметр сушественно снижается. Отметим, что подобная оценка параметра $\kappa$ для одной из точек, а именно для точки максимального отклонения экспериментального значения полного сечения от фитирования на СМ, соответствуюшей значению энергии $\langle\sqrt{S}\rangle=65.8$ ГэВ, получена в работе [13]. Однако этому 
предшествовало упрошение полученного выражения для полного сечения (точнее, отношения полного сечения реакции к сечению “чистой электродинамики", которое и являлось предметом анализа). Упрощение заключалось в предположении, что параметр $\kappa S$ по малости не должен превосходить величину константы $g_{\mathrm{V}}$, поскольку КАВ может внести лиш малую поправку в сечение реакции. Соответственно степени $\kappa S$ более высоких порядков малости, нежели степень $g_{\mathrm{V}}$ в СМ, были опушены. Полученное численное значение $\kappa S=0.33 \pm 0.32$ для $\langle\sqrt{S}\rangle=65.8$ ГэВ дает следуюшее значение параметра:

$$
\begin{aligned}
& \kappa=0.76 \pm 0.74 \times 10^{-6} \Gamma_{\ni} \mathrm{B}^{-2}, \\
& 82 \Gamma_{\ni} \mathrm{B} \leqslant \kappa^{-1 / 2} \leqslant 752 \text { ГэВ. }
\end{aligned}
$$

Отметим также, что значения параметра КАВ вполне согласуются с результатами работы [8], где получены ограничения параметра $\Lambda_{l}$, для значения сечения при $S \approx$ 2108 ГэВ ${ }^{2}$ составляющие $\Lambda_{l}>96$ ГэВ. Из данных для асимметрии этой реакции при $S \approx 1800$ ГэВ ${ }^{2}$ следует ограничение $\Lambda_{l}>108$ ГэВ.

Резюмируя приведенные выше результаты, можно предположить, что полученное численное значение (12) наилучшим образом оценивает параметр возможного КАВ для данной области энергий в реакции по электрон-позитронной аннигиляции в мюон-антимюонную пару.

Благодарности. В заключение автор выражает признательность А. А. Богушу и И. С. Сацункевичу за руководство и помощь, оказанные при выполнении и оформлении данной работы, а также благодарит за поддержку Белорусский республиканский фонд фундаментальных исследований. Автор также выражает благодарность региональному бюро ЮНЕСКО по научному сотрудничеству в Европе за поддержку Международной школы физики им. В. А. Фока.

\section{Список литературы}

[1] Я. Б. Зельдович. ЖЭТФ. 1957. Т. 33. С. 1531.

[2] Я. Б. Зельдович, А. М. Переломов. ЖЭТФ. 1960. Т. 39. С. 1115.

[3] N. Dombey, A. D. Kennedy. Phys. Lett. B. 1980. V. 91. № 3,4. P. 428.

[4] H. Czyz, K. Kolodziej, M. Zralek, P. Cristowa. Can. J. Phys. 1988. V. 66. P. 132.

[5] M. J. Musolf, B. R. Holstein. Phys. Rev. D. 1991. V. 66. P. 2956.

[6] V. V. Flambaum, I. B. Khriplovich, O. P. Sushkov. Phys. Lett. 1984. V. 66. P. 367.

[7] T. A. Góngora, R. G. Stuart. Z. Phys. C. 1992. V. 55. P. 101.

[8] В. В. Гилевский, И. С. Сачункевич. ЯФ. 1997. Т. 60. С. 910.

[9] C. S. Wood, S. C. Bennett, D. Cho et al. Science. 1997. V. 275. P. 1759.

[10] R. Hasty, A. M. Hawthorne-Allen, T. Averett et al. (SAMPLE Collaboration). Science. 2000. V. 290. P. 2117.

[11] M. Acciarri et al. (The L3 Collaboration). Phys. Lett. B. 1996. V. 374. P. 331.

[12] D. E. Groom et al. (Particle Data Group). Eur. J. Phys. C. 2002. V. 15. P. 21.

[13] В. В. Махнач. Вести НАНБ. Сер. физ-мат. наук. 2001. Т. 4. С. 68. 